ARTICLE

DOI: $10.1038 /$ s41467-017-01765-0

\title{
Nanoscale tomography reveals the deactivation of automotive copper-exchanged zeolite catalysts
}

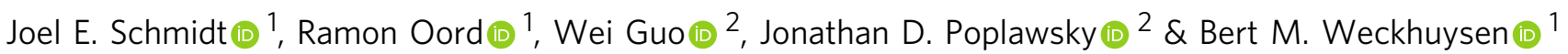

Copper-exchanged zeolite chabazite (Cu-SSZ-13) was recently commercialized for the selective catalytic reduction of $\mathrm{NO}_{x}$ with ammonia in vehicle emissions as it exhibits superior reaction performance and stability compared to all other catalysts, notably Cu-ZSM-5. Herein, the 3D distributions of $\mathrm{Cu}$ as well as framework elements ( $\mathrm{Al}, \mathrm{O}, \mathrm{Si}$ ) in both fresh and aged $\mathrm{Cu}-\mathrm{SSZ}-13$ and $\mathrm{Cu}-\mathrm{ZSM}-5$ are determined with nanometer resolution using atom probe tomography (APT), and correlated with catalytic activity and other characterizations. Both fresh catalysts contain a heterogeneous $\mathrm{Cu}$ distribution, which is only identified due to the single atom sensitivity of APT. After the industry standard 135,000 mile simulation, Cu-SSZ13 shows $\mathrm{Cu}$ and $\mathrm{Al}$ clustering, whereas $\mathrm{Cu}-\mathrm{ZSM}-5$ is characterized by severe $\mathrm{Cu}$ and $\mathrm{Al}$ aggregation into a copper aluminate phase $\left(\mathrm{CuAl}_{2} \mathrm{O}_{4}\right.$ spinel). The application of APT as a sensitive and local characterization method provides identification of nanometer scale heterogeneities that lead to catalytic activity and material deactivation.

\footnotetext{
${ }^{1}$ Debye Institute for Nanomaterials Science, Utrecht University, Universiteitsweg 99, 3584 CG Utrecht, The Netherlands. ${ }^{2}$ Center for Nanophase Materials Sciences, Oak Ridge National Laboratory, Oak Ridge, TN 37831, USA. Correspondence and requests for materials should be addressed to J.D.P. (email: poplawskyjd@ornl.gov) or to B.M.W. (email: B.M.Weckhuysen@uu.nl)
} 
$\mathrm{n}$ modern diesel vehicles, meeting the mandated goals of decreased fuel consumption, particulate and $\mathrm{NO}_{X}$ emissions requires end-of-tailpipe technologies due to their interconnected nature ${ }^{1}$. Although several strategies exist for mobile $\mathrm{NO}_{X}$ reduction, by far the most effective is ammonia selective catalytic reduction $\left(\mathrm{NH}_{3}\right.$-SCR), with urea serving as a source of the ammonia reductant, and the reaction occurring over a copper $(\mathrm{Cu})$-exchanged zeolite catalyst ${ }^{2-4}$. Mobile SCR catalysts face formidable requirements including stable operation across low (engine start) and high (hydrocarbon contaminant burn off) temperature regimes, resistance to poisoning and long lifetimes ${ }^{5}$. $\mathrm{Cu}$-exchanged zeolite ZSM-5 (further denoted as Cu-ZSM-5) was first described for SCR in $1986^{6}$, but is unable to meet lifetime requirements due to deactivation under tailpipe conditions ${ }^{7-9}$. A true success story of recent zeolite catalysis was the discovery of $\mathrm{Cu}$-exchanged zeolite SSZ-13 (further denoted as Cu-SSZ-13) for mobile $\mathrm{NO}_{X}$ SCR with ammonia (further denoted as $\operatorname{deNO}_{X}$ ) in the mid-2000s, followed by its rapid commercialization in 2010, due to its high activity and stability $5,10-12$.

Zeolite SSZ-13 is a small-pore zeolite with the chabazite (CHA) framework, containing cages that are limited by 8 -membered ring (8 MR) windows $(\sim 3.7 \AA)$, and the silicoaluminophosphate composition of this structure, i.e., SAPO-34, is currently applied commercially for the methanol-to-hydrocarbons (MTH) reaction $^{13}$. The superior performance of Cu-SSZ-13 as a mobile $\operatorname{deNO}_{X}$ catalyst is ascribed to its small pores preventing the admission of contaminants and Al migration, the double sixmembered rings (d6r's) serving as the preferential $\mathrm{Cu}$ exchange site, and the intrinsic stability of the framework structure ${ }^{14-29}$. Deactivation of $\mathrm{Cu}$-exchanged zeolite catalysts has been studied by numerous characterization techniques, and is thought to occur through a combination of loss of $\mathrm{Al}$ from the zeolite framework and $\mathrm{Cu}$ migration to form $\mathrm{Cu}$ oxides and $\mathrm{Cu}$ aluminate species, similar to a $\mathrm{CuAl}_{2} \mathrm{O}_{4}$ spinel phase $\mathrm{e}^{5,14,15,21,24,30-34}$. For Cu-ZSM5 , it is known that the deactivated material retains crystallinity, despite loss of framework $\mathrm{Al}$ which is able to move through the 10 MR pores, as well as migration and sintering of $\mathrm{Cu}$, whereas $\mathrm{Cu}$ SSZ-13 is not deactivated under identical conditions $7,8,23,27,34-36$ However, there still remain significant gaps in our understanding of the exact deactivation mechanisms in these materials due to the small size of deactivating species, which makes them invisible to many techniques, e.g., X-ray diffraction, and the presence of paramagnetic $\mathrm{Cu}$ which can interfere with locally sensitive techniques, e.g., NMR. Therefore, it is necessary to reconstruct the distribution of single atoms in $3 \mathrm{D}$ to gain a complete picture of deactivation.

Here, we show the material changes upon aging by creating atom-by-atom reconstructions of both fresh and aged, industrially relevant zeolite catalysts using atom probe tomography (APT). Zeolites Cu-ZSM-5 and Cu-SSZ-13 are aged using the industry standard protocol to simulate 135,000 miles of vehicle use, and characterized using the conventional techniques of powder X-ray diffraction (XRD), ammonia temperatureprogrammed desorption $\left(\mathrm{NH}_{3}-\mathrm{TPD}\right)$, UV-Vis-NIR diffuse reflectance spectroscopy (UV-Vis-NIR-DRS) and $\operatorname{deNO}_{X}$ reaction testing ${ }^{28}$. The distributions of $\mathrm{Al}$ and $\mathrm{Cu}$ are mapped with nanometer resolution in $3 \mathrm{D}$ using APT. Heterogeneities in both the $\mathrm{Cu}$ and $\mathrm{Al}$ distributions are found in all four catalysts (i.e., fresh and aged $\mathrm{Cu}-\mathrm{ZSM}-5$ and $\mathrm{Cu}-\mathrm{SSZ}-13$ ), and the significant changes between the fresh and aged materials can account for the observed differences in $\operatorname{deNO}_{X}$ reactivity, as is schematically shown in Fig. 1.

\section{Results}

Catalyst characterization and reactivity. The four $\mathrm{Cu}$-exchanged zeolite materials used in this work are typical for commercially relevant $\operatorname{deNO}_{X}$ catalysts ${ }^{12,28}$, with details on their preparation and characterization given in the Methods and Supplementary Methods. The results of the $\mathrm{deNO}_{X}$ reaction testing are given in
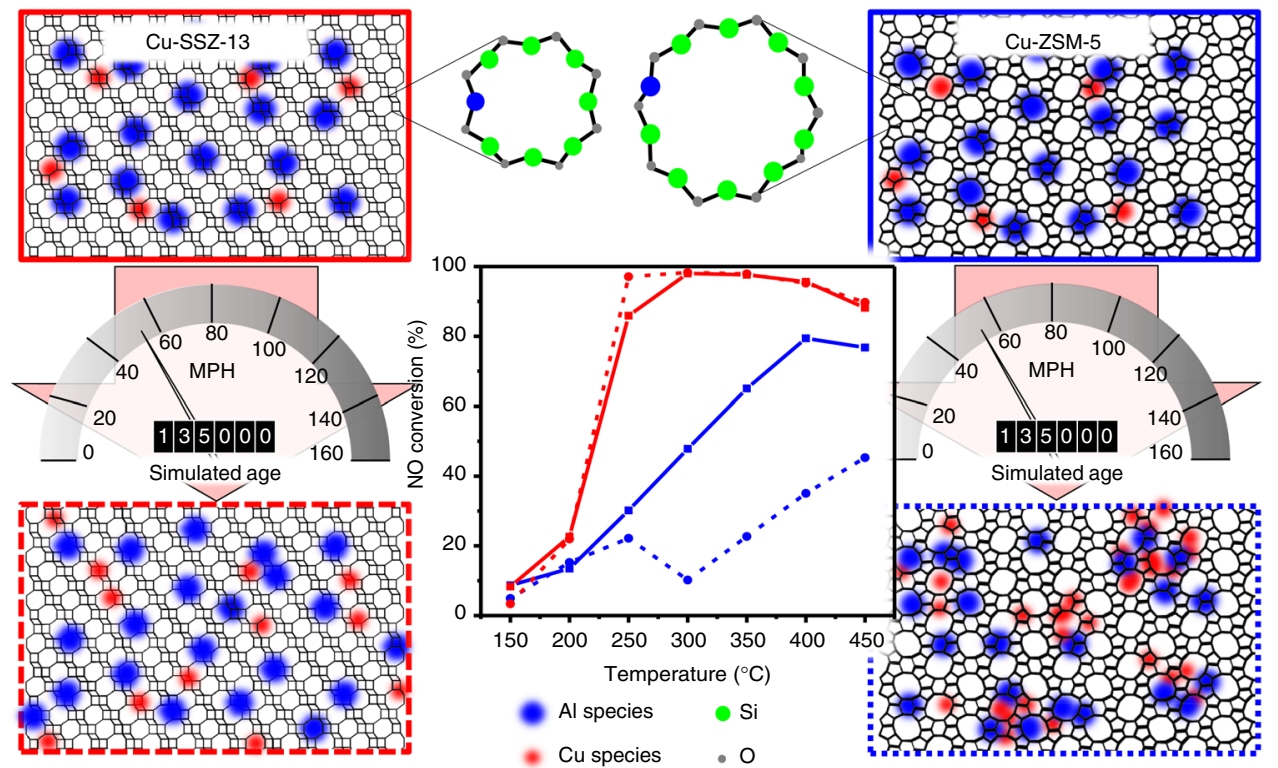

Fig. 1 Reaction testing and overview of experimental findings. The center graph shows the NO conversion for the ammonia selective catalytic reduction $\left(\mathrm{NH}_{3}-\mathrm{SCR}\right)$ reaction testing results for the four $\mathrm{Cu}$-exchanged zeolite materials under study, the traces correspond to the borders of the four schematics. The feed consists of $1000 \mathrm{ppm} \mathrm{NO}, 1000 \mathrm{ppm} \mathrm{NH}_{3}, 5 \% \mathrm{O}_{2}$, balanced by He. The data have been measured in steady-state mode $\left(\mathrm{GHSV}=100,000 \mathrm{~h}^{-1}\right.$ ). The panels at the corners give a 2D schematic overview of the results of this study with regards to the distribution of $\mathrm{Al}$ and $\mathrm{Cu}$ in $\mathrm{Cu}$-SSZ-13 and $\mathrm{Cu}$-ZSM5, which are distributed slightly heterogeneously in fresh $\mathrm{Cu}-\mathrm{SSZ}-13$ and $\mathrm{Cu}-\mathrm{ZSM}-5$. After the simulated 135,000 mile aging procedure the Cu in Cu-SSZ-13 is found to migrate somewhat, as the schematic shows, though the material still retains good catalytic activity. In Cu-ZSM-5, the simulated aging causes significant migration of $\mathrm{Cu}$ and $\mathrm{Al}$ into stoichiometric copper aluminate species. One of the significant reasons that Cu-ZSM- 5 is more damaged is that both $\mathrm{Cu}$ and $\mathrm{Al}$ species are able to migrate through its larger rings, whereas the small rings in Cu-SSZ-13 prevent Al migration, limiting damage to the material 
Fig. 1, and show that zeolite Cu-SSZ-13 retains its activity even after aging, whereas zeolite Cu-ZSM-5 shows a significant reduction in deNO ${ }_{X}$ activity after aging, in line with the results of other studies ${ }^{5,7-12}$. The XRD patterns of the fresh and aged $\mathrm{Cu}-$ ZSM-5 and Cu-SSZ-13 materials are shown in Supplementary Figs. 1 and 2, and in all four samples no significant framework destruction or peaks associated with $\mathrm{Cu}$ oxides or $\mathrm{CuAl}_{2} \mathrm{O}_{4}$ spinel could be observed, meaning that the zeolite frameworks remain intact after aging and that $\mathrm{Cu}$ is well-dispersed and is not agglomerated, at least at length scales that the XRD method is capable of detecting. The inability to detect material changes with $\mathrm{XRD}$ reinforces the need for advanced characterization techniques to truly elucidate deactivation phenomena. UV-Vis-NIRDRS measurements show a significant difference between the two $\mathrm{Cu}$-exchanged zeolite materials, with further changes after aging that indicate possible $\mathrm{CuO}$ and $\mathrm{CuAl}_{2} \mathrm{O}_{4}$ spinel species, shown in Supplementary Figs. 3 and 4, along with a detailed explanation of the interpretation of the spectra in the Supplementary Methods. $\mathrm{NH}_{3}$-TPD (Supplementary Methods; Supplementary Fig. 5) shows the expected changes with aging, with a decrease in strong acid sites attributed to framework $\mathrm{Al}$ removal, and $\mathrm{Cu}$ ions present in all samples ${ }^{18}$. Although these bulk techniques indicate changes to the material after aging, they offer a global view of a nanoscopic phenomena, motivating our study.

An unanswered question, critical to understanding the deactivation of these catalysts, is the fate of $\mathrm{Al}$ and $\mathrm{Cu}$ after removal from the framework. Chemically or spatially resolved insights regarding the deactivation of $\mathrm{Cu}$-exchanged zeolites have been gained using X-ray absorption fine structure (XAFS), transmission electron microscopy (TEM), scanning TEM
(STEM), STEM electron dispersion spectroscopy (STEM-EDS), and X-ray photoelectron spectroscopy (XPS), but these techniques suffer from significant drawbacks including the inability to detect isolated ions, as well as offering only $2 \mathrm{D}$ information or bulk averages ${ }^{14,24,28,30-34,37,38}$. To gain a more complete picture of the mechanism of deactivation in these materials, and establish the distribution of both $\mathrm{Cu}$ and framework elements (e.g., $\mathrm{Al}$ and $\mathrm{Si}$ ), we studied all four samples using APT, and differences in the elemental distributions are correlated with both spectroscopy and reactivity. A recent study on Fe-SSZ-13 used APT to characterize the material before and after aging, with the conclusion that the Fe was randomly distributed and not clustered in either sample, but the APT results were in disagreement with the findings of TEM/EDS, showing the need for a more comprehensive study of the commercialized Cu-SSZ-13 catalyst ${ }^{39}$. A potential challenge in studying Fe-SSZ-13 is that there is overlap between the primary $\mathrm{Fe}\left({ }^{56} \mathrm{Fe}^{2+}\right)$ and $\mathrm{Si}\left({ }^{28} \mathrm{Si}^{+}\right)$peaks in the mass spectrum, potentially complicating the quantification and subsequent analysis, but no similar overlap is encountered in the present work with $\mathrm{Cu}$. Full details of APT sample preparation, experiment and data analysis are given in the Methods and Supplementary Methods, and SEM images of both catalysts before and after being fashioned into needles for APT analysis are shown in Supplementary Fig. 6. When the needles were reconstructed, compositional heterogeneities were found that spanned a range of length scales, which can account for the differences in $\mathrm{deNO}_{X}$ reaction behavior. The following sections will be structured by first discussing Cu-ZSM- 5 and then Cu-SSZ13, followed by a comparison of the two materials, and Supplementary Table 1 lists all samples along with the
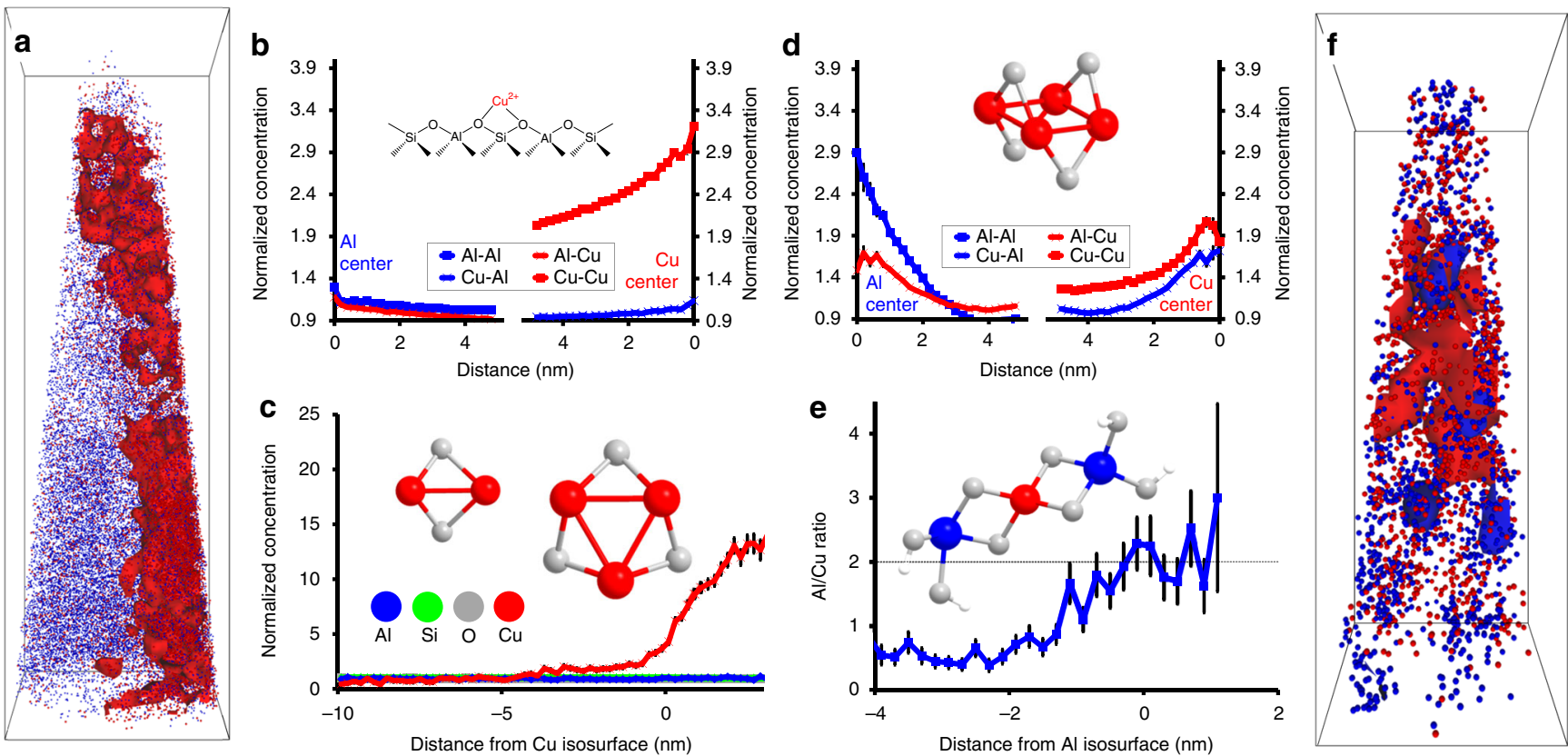

Fig. 2 Overview of atom probe tomography results for fresh and aged Cu-ZSM-5. a Reconstructed needle of fresh Cu-ZSM-5 (1, full results are in Supplementary Fig. 22) with $\mathrm{Cu}$ (red) and $\mathrm{Al}$ (blue) ions shown and $1.8 \% \mathrm{Cu}$ isoconcentration surfaces shown, this is also provided as Supplementary Movie 1. Bonding box dimensions are $40 \times 43 \times 140 \mathrm{~nm}^{3}$. b Radial distribution functions (RDFs) in fresh Cu-ZSM-5 for $\mathrm{Al}$ and $\mathrm{Cu}$ centers. Strong Cu-Cu affinity is indicated, as well as much weaker, but still significant affinities between all other species. An Al-Cu affinity is expected due to $\mathrm{Cu}$ exchanging onto paired Al sites, as indicated in the schematic. c Normalized compositional histogram across the 1.8\% Cu isoconcentration surfaces in fresh Cu-ZSM-5, with the structures of potential $\mathrm{CuO}$ species shown. $\mathbf{d}$ RDFs in aged Cu-ZSM-5 (3, full results are in Supplementary Fig. 26) for Al and Cu centers, with the structure of a potential $\mathrm{CuO}$ species shown. Strong affinities are indicated between all species, pointing to the formation of copper aluminate domains after aging due to $\mathrm{Cu}-\mathrm{Al}$ aggregation. e $\mathrm{Al} / \mathrm{Cu}$ ratio across the $8 \% \mathrm{Al}$ isoconcentration surfaces in aged $\mathrm{Cu}-\mathrm{ZSM}-5$, the stoichiometry of $\mathrm{Al} / \mathrm{Cu}=2$ is consistent with $\mathrm{CuAl}_{2} \mathrm{O}_{4}$ spinel, and the O/Cu and O/AI values are also stoichiometric, see Supplementary Fig. 27. f Reconstructed needle of aged Cu-ZSM-5 (3) with $5 \% \mathrm{Cu}$ and $8 \% \mathrm{Al}$ isoconcentration surfaces shown, this is also included as Supplementary Movie 3. Bonding box dimensions are $15 \times 15 \times 43 \mathrm{~nm}{ }^{3}$. All error bars were calculated from counting statistics using the method described in ref. 55 

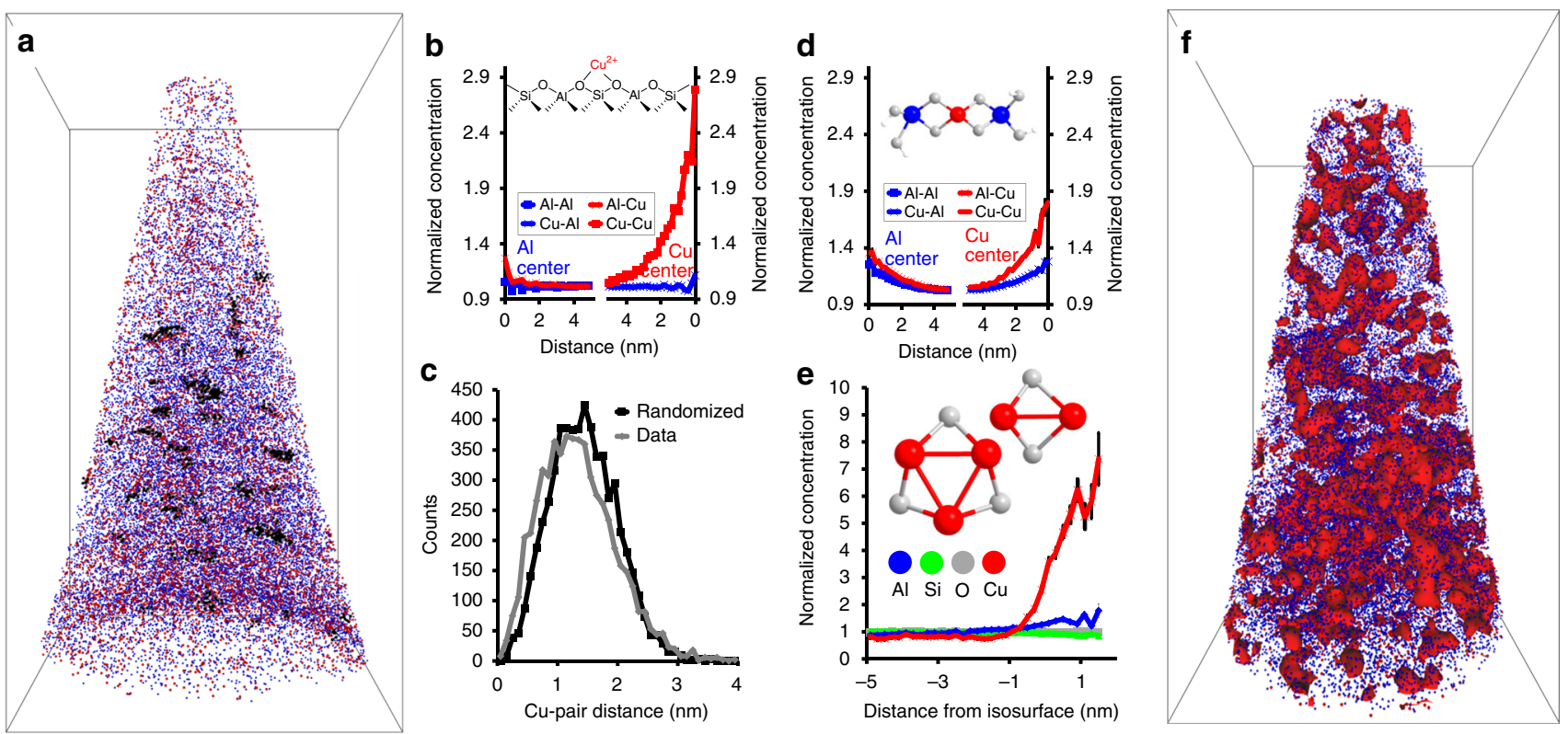

Fig. 3 Overview of atom probe tomography results for fresh and aged CU-SSZ-13. a Reconstructed needle of fresh Cu-SSZ-13 (4, full results are in Supplementary Fig. 28) with $\mathrm{Cu}$ (red) and Al (blue) ions shown with Cu clusters overlaid in black, this is also included as Supplementary Movies 4 and 5. Bonding box dimensions are $63 \times 67 \times 115 \mathrm{~nm}^{3}$. b Radial distribution functions (RDFs) in fresh Cu-SSZ-13 for Al and Cu centers. Al-Cu affinity is indicated, which would be expected due to $\mathrm{Cu}$ exchanging onto paired Al sites, as indicated in the schematic. c Nearest neighbor distribution for fresh Cu-SSZ-13 for Cu showing a significant deviation from a random distribution and indicating the presence of Cu clusters. d RDFs in aged Cu-SSZ-13 (6, full results are in Supplementary Fig. 32) for Al and Cu centers. Strong affinities are indicated between all species, pointing to the migration and aggregation of $\mathrm{Cu}$ with aging, and a $\mathrm{Cu}$ aluminate species is shown, though it was not quantitatively identified. e Normalized compositional histogram across $1.4 \% \mathrm{Cu}$ isoconcentration surfaces in aged Cu-SSZ-13, with potential CuO species shown. f Reconstructed needle of aged Cu-SSZ-13 (6) with 1.4\% Cu isoconcentration surfaces shown, this is also included as Supplementary Movies 8 and 9 . Bonding box dimensions are $49 \times 52 \times 90 \mathrm{~nm}^{3}$. All error bars were calculated from counting statistics using the method described in ref. 55

compositions determined using APT, and a complete discussion of the APT analysis for each material is in the Supplementary Methods and Supplementary Figs. 7-36. In addition, Supplementary Movies 1-10 show each needle to allow for easy 3D visualization of the ion distributions and features of interest.

Atom probe tomography of fresh and aged zeolite Cu-ZSM-5. Two needles of fresh Cu-ZSM-5 and one of aged Cu-ZSM-5 were successfully reconstructed as this material was especially prone to failure, with images of the fresh and aged materials shown in Fig. 2 and Supplementary Fig. 19; in all subsequent discussions of the fresh and aged materials, samples 1 to 3 , complete APT results are in Fig. 2 and Supplementary Figs. $22-27$ as well as Supplementary Movies 1-3. In fresh Cu-ZSM-5, 1, the Cu segregation was easily isolated using a 1.8 atomic $\% \mathrm{Cu}$ isoconcentration surface (bulk $\mathrm{Cu}$ content is 0.4 atomic \%, all subsequent compositions are in atomic percent and will be referred to by $\%$ for brevity). The compositional histogram across this isoconcentration surface shows a rapid increase in the $\mathrm{Cu}$ content to nearly 15 times its bulk normalized concentration. Interestingly, although the $\mathrm{Cu}$ content rises markedly across this region, the $\mathrm{Al}$ content only changes slightly, indicating that the heterogeneous $\mathrm{Cu}$ distribution is possibly caused by preferential material accessibility during the exchange. This $\mathrm{Cu}$ segregation is reflected in the $\mathrm{Cu}$ nearest neighbor distribution (NND) as well as the $\mathrm{Cu}$ radial distribution function (RDF). The Al NND for $\mathbf{1}$ also shows a small deviation from the normal distribution, which was studied using cluster analysis, with the identification of significant $\mathrm{Al}$ clusters shown in Supplementary Fig. 22. In our previous work using APT to study large, carefully prepared crystals of ZSM-5, the $\mathrm{Al}$ distribution was random in the fresh material, so finding clustered $\mathrm{Al}$ in the commercial material highlights the imperfections found in industrial catalysts compared to model systems ${ }^{40}$. This assertion is supported by the recent application of advanced microscale and nanoscale characterization techniques, which have revealed that small, industrial zeolite crystals can be quite heterogeneous, and that the results of bulk studies give an ensemble average from a diverse population of crystals ${ }^{41-45}$. The second needle of fresh Cu-ZSM-5, 2, also had $\mathrm{Cu}$ segregation, which is reflected in the NND and RDF. This segregation was isolated using both a $2.7 \% \mathrm{Cu}$ isoconcentration surface and $\mathrm{Cu}$ cluster analysis as the regions could be described by either method. This needle had a homogeneous $\mathrm{Al}$ distribution, and the differences between the two needles from the same material reinforce the imperfect nature of industrial zeolite crystals, and it is possible that the heterogeneity of the material contributes to its lower activity and stability.

After aging, significant changes occurred in both the $\mathrm{Cu}$ and $\mathrm{Al}$ distributions, which are immediately apparent from ion distribution maps in Fig. 2f and Supplementary Fig. 19, with complete characterizations of sample 3 in Supplementary Figs. 26 and 27 and Supplementary Movie 3. The NND for Al shows a significant deviation from a random distribution in this material, and the $\mathrm{Al}$ $\mathrm{RDF}$ shows that there is an $\mathrm{Al}-\mathrm{Al}$ affinity, as well as an $\mathrm{Al}-\mathrm{Cu}$ affinity, which are caused by a $\mathrm{Cu}-\mathrm{Al}$ aggregation in the sample. An $8 \% \mathrm{Al}$ isoconcentration surface was analyzed (bulk $\mathrm{Al}$ content is $3.7 \%$ ), and its relevance to understanding deactivation is evident when the $\mathrm{Al} / \mathrm{Cu}$ ratio is considered. Across the $8 \% \mathrm{Al}$ isoconcentration surface the $\mathrm{Al} / \mathrm{Cu}$ ratio increases to $\sim 2$, which matches the stoichiometry of $\mathrm{Cu}$ aluminate, a $\mathrm{CuAl}_{2} \mathrm{O}_{4}$ spinel species, which has long been regarded as one of the species that forms upon aging of these materials. Previously, it was only identified by bulk analysis, and here is shown as spatially isolated 
nanoscale features in $3 \mathrm{D}^{14,24,31-34}$. In addition, the $\mathrm{O} / \mathrm{Cu}$ and $\mathrm{O} /$ $\mathrm{Al}$ values, shown in Supplementary Fig. 27, agree with their stoichiometric values inside these isoconcentration surfaces, further supporting the identification of $\mathrm{CuAl}_{2} \mathrm{O}_{4}$ spinel within the APT data. Upon further examination of the UV-Vis-NIRDRS spectra (Supplementary Figs. 3 and 4), it is likely that changes upon aging are due to the presence of $\mathrm{CuAl}_{2} \mathrm{O}_{4}$ spinel and $\mathrm{CuO}$ species, though these phases are difficult to verify by spectroscopy alone due to many overlapping peaks and the influence of domain size on the peak shift, underscoring the need for advanced characterization methods to understand the formation and presence of deactivating phases. The proportion of the material contained in these $\mathrm{Cu}$ aluminate regions was assessed from the APT data and found to include $\sim 20 \%$ of all $\mathrm{Cu}$ and $\sim 35 \%$ of all Al (further discussed in the Supplementary Fig. 26 caption), providing a rough estimate of the loss of active sites. Therefore, we have been able to demonstrate a $\mathrm{CuAl}_{2} \mathrm{O}_{4}$ stoichiometry in a nanoscale region and determine the proportion of material that has transformed into this phase after aging. Data were successfully collected from a second needle of aged $\mathrm{Cu}-$ ZSM-5, and the results are consistent with those of needle 3, which can be found in the Supplementary Methods and Supplementary Figs. 35 and 36, though these data were not included in the main manuscript due to significant Ga implantation.

\section{Atom probe tomography of fresh and aged zeolite Cu-SSZ-13.} Two needles of fresh zeolite Cu-SSZ-13 were measured, with all discussed results for samples $\mathbf{4}$ and $\mathbf{5}$ shown in Fig. 3 and Supplementary Figs. 28-31 and ion maps within Supplementary Fig. 20 as well as Supplementary Movies $4-7$, with only minor heterogeneities apparent from visual inspection. The Al distribution was random as no deviation was observed from the NND; this contrasts with the Cu-ZSM-5 and may be due to the different preparation methods of the zeolites. Finding a random $\mathrm{Al}$ distribution suggests that a $\mathrm{Cu}$ exchange is unlikely to cause $\mathrm{Al}$ segregation, so the observation of a heterogeneous $\mathrm{Al}$ distribution in fresh Cu-ZSM-5 is most likely not caused by ion exchange. The $\mathrm{RDF}$ for $\mathrm{Al}$ shows an $\mathrm{Al}-\mathrm{Cu}$ affinity, though this would be expected as $\mathrm{Al}$ serves as the exchange site for $\mathrm{Cu}$, shown schematically in Fig. 3b, highlighting the sensitivity of APT. The $\mathrm{Cu}$ distribution is not homogeneous, which is seen in the $\mathrm{Cu}$ NNDs for both needles, and the $\mathrm{Cu}$ RDFs indicate that there is a $\mathrm{Cu}-\mathrm{Cu}$ affinity. Cluster analysis for $\mathrm{Cu}$ in these fresh materials gave statistically significant $\mathrm{Cu}$ clustering in both needles, and it was found that looking at the fourth nearest neighbor $(\mathrm{O}=4)$ led to the best separation of $\mathrm{Cu}$ clusters (further discussed in the Supplementary Methods ${ }^{46,47}$. The cluster analysis demonstrates that even in the fresh $\mathrm{Cu}-\mathrm{SSZ}-13$, which has only isolated $\mathrm{Cu}$ ions based the UV-Vis-NIR-DRS spectrum, a non-random $\mathrm{Cu}$ dispersion exists. It is known in small-pore zeolites that conventional aqueous ion exchange of $\mathrm{Cu}$ can be unpredictable and more challenging than larger pore materials, and this non-random $\mathrm{Cu}$ dispersion may be the result of this known difficulty ${ }^{48,49}$. This finding shows the $\mathrm{Cu}$ dispersion can be heterogeneous, though still as spectroscopically isolated $\mathrm{Cu}$, giving a highly active catalyst. In addition, it highlights the power of APT to probe these materials as no other techniques can resolve isolated $\mathrm{Cu}$ ions in

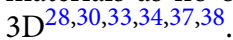

After aging, the Cu-SSZ-13 still shows good deNO ${ }_{X}$ activity, with some changes in the UV-Vis-NIR-DRS spectrum, corresponding to the formation of $\mathrm{CuO}$, though these species must be small as they are not detected using XRD, and potential $\mathrm{CuO}$ species are shown in Fig. 3e. Complete APT characterization of samples 6 and 7 is in Fig. 3, Supplementary Figs. 32-34 and
Supplementary Movies 8-10. The NNDs for Al show a small deviation from a random distribution, indicating that aging has caused the removal of some $\mathrm{Al}$ from framework positions, and aggregation into $\mathrm{Al}$ and $\mathrm{Cu}$-rich regions is evident from the RDFs. Although this does indicate some material destruction, it is less significant than in Cu-ZSM-5, which is reflected in the better stability and activity of the catalyst. The $\mathrm{Cu}$ distribution is also heterogeneous, easily shown in the $\mathrm{Cu}$ NNDs, and the Cu RDFs show $\mathrm{Cu}-\mathrm{Cu}$ and $\mathrm{Cu}-\mathrm{Al}$ affinities, a difference from what was found in the fresh material where no $\mathrm{Cu}-\mathrm{Al}$ affinity was observed, and indicates some $\mathrm{Cu}-\mathrm{Al}$ aggregation in the sample. Therefore, even though no significant formation of $\mathrm{Cu}$ aluminate domains is indicated in the aged material, a clear affinity of $\mathrm{Cu}$ for $\mathrm{Al}$ is demonstrated by APT. The Cu was significantly segregated such that $\mathrm{Cu}$-rich regions could be easily identified using isoconcentration surface analysis and compositional histograms. Although there is a less pronounced increase in $\mathrm{Al}$ in the $\mathrm{Cu}$-rich regions compared with aged $\mathrm{Cu}-\mathrm{ZSM}-5$, it is still present, consistent with limited $\mathrm{Cu}$ and $\mathrm{Al}$ migration with aging. These observations agree with the known stabilizing influence of $\mathrm{Cu}$ in SSZ-13, which have been observed since the earliest reports on the material, and is thought to be caused by the $\mathrm{Cu}(\mathrm{II})$ exchanging onto paired $\mathrm{Al}$ sites, which stabilizes the material by preventing hydrolysis of the $\mathrm{Al}-\mathrm{O}$ bonds ${ }^{10}$. Overall, this leads to limited material destruction and explains the remarkable stability of the material under demanding conditions.

\section{Discussion}

The distributions of $\mathrm{Cu}$ and $\mathrm{Al}$ in the fresh and aged Cu-ZSM-5 and $\mathrm{Cu}-\mathrm{SSZ}-13$ materials help to explain the deNO $\mathrm{N}_{X}$ activity, shown in Fig. 1, as APT can detect features that are not observable by any other characterization techniques. A comparison of $20 \times 20 \times 20 \mathrm{~nm}$ regions from the four zeolite samples is shown in Fig. 4. Fresh zeolite $\mathrm{Cu}-\mathrm{SSZ}-13$ has a random $\mathrm{Al}$ distribution, but in fresh zeolite Cu-ZSM-5 the Al distribution is slightly
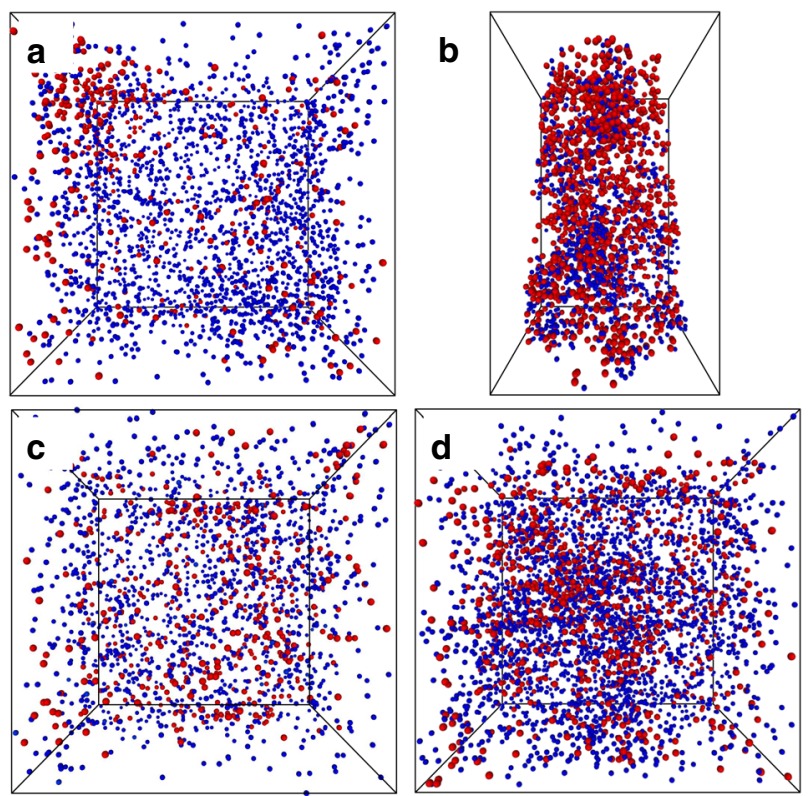

Fig. 4 Selection of 3D atom probe tomography data for comparison. $20 \times$ $20 \times 20 \mathrm{~nm}^{3}$ cubic regions selected from each sample to give a closer comparison of the $\mathrm{Cu}$ (red) and $\mathrm{Al}$ (blue) distributions, with locations shown in Supplementary Fig. 21, and a schematic overview of these results is given in Fig. 1. a Fresh Cu-ZSM-5 (1), b aged Cu-ZSM-5 (3, the bounding box is $20 \times 13 \times 13 \mathrm{~nm}^{3}$ as this needle was smaller), c fresh Cu-SSZ-13 (4), and $\mathbf{d}$ aged Cu-SSZ-13 (7) 
heterogeneous, likely reflecting the different zeolite synthesis procedures, but highlighting the importance of understanding that real-life catalyst materials may be prepared under non-ideal conditions to satisfy economic requirements, leading to structural and compositional imperfections in the materials that are not encountered under more time consuming, small-scale laboratory preparation procedures, and this has been recently shown by several groups using a variety of techniques ${ }^{41-45}$. Both materials were $\mathrm{Cu}$-exchanged using conventional aqueous procedures, followed by calcination. Previous characterization studies have shown that this results in well-dispersed $\mathrm{Cu}$ ions, though it is known to be more difficult to exchange $\mathrm{Cu}$ in small-pore materials $^{50}$. However, heterogeneities found in both materials from analysis of the APT data show that $\mathrm{Cu}$-rich regions tend to form. It is possible that the lower activity of the fresh zeolite Cu-ZSM-5 compared to the fresh zeolite Cu-SSZ-13 can be at least partially attributed to differences in the $\mathrm{Cu}$ dispersion, but we do not currently have sufficient data to clearly demonstrate this hypothesis. A recent experimental and theoretical report has demonstrated that $\mathrm{NO}_{X}$ SCR with Cu-SSZ-13 falls outside the conventional boundaries of homogeneous or heterogeneous catalysis as the reaction exhibits a density-dependent interaction of multiple ionically tethered single sites ${ }^{51}$. Of importance to the present findings is that optimization of the $\mathrm{Cu}$ spatial distribution or mobility is vital to improving low-temperature performance, as $\mathrm{Cu}$ ions are found to have a maximum diffusion distance of $\sim 0.9 \mathrm{~nm}$ due to electrostatic tethering. Although a random $\mathrm{Cu}$ distribution is normally assumed, we have shown in fresh $\mathrm{Cu}$ SSZ-13 that this is not the case, as $\mathrm{Cu}$-rich regions have been identified (Fig. 3), which may have important implications for forming dynamic multinuclear sites, and may also lead for highly heterogeneous reaction behavior within a single zeolite crystal.

After aging according to an industrially relevant methodology, the contrasts between the zeolite frameworks are wellpronounced. In the aged zeolite $\mathrm{Cu}-\mathrm{SSZ}-13$, APT shows $\mathrm{Cu}$ and $\mathrm{Al}$ clustering, but to a limited degree. This is not strongly reflected in the deNO ${ }_{X}$ activity, and it is possible that this is due to the high conversions as well as the temperatures examined ${ }^{50}$. The changes in the UV-Vis-NIR-DRS spectrum after aging indicate the possible formation of $\mathrm{CuO}$ species, which is consistent with the change in the $\mathrm{Cu}$ distribution as probed with APT, providing important secondary confirmation as the $\mathrm{CuO}$ region is overlapped with LMCT transitions of $\mathrm{Cu}^{2+}$. Significant changes for aged zeolite Cu-ZSM-5 were evident from the $\operatorname{deNO}_{X}$ activity, UV-Vis-NIR-DRS spectroscopy as well as APT. The APT isoconcentration surface analysis shows that both the $\mathrm{Cu}$ and $\mathrm{Al}$ migrate and segregate, and the formation of $\mathrm{Cu}$-rich regions, $\mathrm{Al}-$ rich regions, as well as $\mathrm{Cu}$ aluminate domains, has been identified. This is an important observation, and one that is not trivial as the paramagnetic $\mathrm{Cu}$ complicates ${ }^{27} \mathrm{Al}$ NMR data collection and quantification to understand changes to the local environment of $\mathrm{Al}$, which are too small to be detected by XRD. We have demonstrated using APT that as the $\mathrm{Al}$ is lost from framework positions it migrates into Al-rich regions, and the formation of $\mathrm{Cu}$ aluminate domains is indicated by the affinity of $\mathrm{Cu}$ for $\mathrm{Al}$, and vice versa, after aging, leading to $\mathrm{Cu}-\mathrm{Al}$ aggregation. In aged zeolite $\mathrm{Cu}-\mathrm{ZSM}-5$ domains of $\mathrm{CuAl}_{2} \mathrm{O}_{4}$ spinel have been mapped in $3 \mathrm{D}$ and their proportion relative to all $\mathrm{Cu}$ and $\mathrm{Al}$ species in the material has been estimated, giving insight into material deactivation. In the $\mathrm{CHA}$ framework the small $8 \mathrm{MR}$ pores are very stable, preventing $\mathrm{Al}$ migration and subsequent deactivating $\mathrm{Al}-\mathrm{Cu}$ clustering to the same extent as is possible in the MFI framework with its larger $10 \mathrm{MR}$ pores, greatly limiting the degradation of this material, as is illustrated in Fig. 1. However, the $\mathrm{CHA}$ pores allow $\mathrm{Cu}$ migration, as recently demonstrated to be vital for low-temperature reactivity, though as there is limited migration of $\mathrm{Al}$, the $\mathrm{Cu}$ remains electrostatically tethered and therefore catalytically active, explaining the retained performance of the material as inactive copper aluminate species are not significantly formed ${ }^{51}$.

In summary, zeolite $\mathrm{Cu}-\mathrm{SSZ}-13$ provides superior catalytic performance, and is importantly able to maintain that performance after a simulated 135,000 mile aging, whereas the deNO ${ }_{X}$ performance of $\mathrm{Cu}-\mathrm{ZSM}-5$ degrades significantly. This difference has been ascribed to framework destruction through the removal of $\mathrm{Al}$, which serves as the exchange site for $\mathrm{Cu}$, causing the formation of $\mathrm{CuO}$ and $\mathrm{CuAl}_{2} \mathrm{O}_{4}$ species, which have been previously identified with bulk characterization methods. Using APT as a sensitive nanoscale chemical imaging method, we have studied the $3 \mathrm{D}$ distribution of $\mathrm{Cu}$, the active site, as well as the inorganic framework elements, in particular $\mathrm{Al}, \mathrm{O}$, and $\mathrm{Si}$, in both zeolites $\mathrm{Cu}-\mathrm{SSZ}-13$ and Cu-ZSM-5. Both fresh catalyst materials contain a heterogeneous $\mathrm{Cu}$ distribution, though to a much greater extent in zeolite $\mathrm{Cu}-\mathrm{ZSM}-5$, which also contains a heterogeneous $\mathrm{Al}$ distribution. After aging, zeolite $\mathrm{Cu}-\mathrm{SSZ}-13$ shows some $\mathrm{Cu}$ and $\mathrm{Al}$ clustering, whereas zeolite $\mathrm{Cu}-\mathrm{ZSM}-5$ shows severe $\mathrm{Cu}$ and $\mathrm{Al}$ aggregation, and the presence of copper aluminate (i.e., a $\mathrm{CuAl}_{2} \mathrm{O}_{4}$ spinel phase) is demonstrated and mapped in 3D. The result of finding nanometer scale heterogeneities that nicely correlate with catalyst activity is only possible due to the application of APT as a powerful local characterization method. The findings of this study further reinforce the fundamental mechanisms behind the stability of the CHA framework under demanding tailpipe reaction conditions.

\section{Methods}

Catalyst materials and aging. The SSZ-13 was prepared in our lab as a commercial version is not available. Hereto, a $25 \%$ solution of the structure directing agent (SDA, N,N,N-trimethyl-1-adamantammonium, Sachem) was added to tetraethylorthosilicate (TEOS, Aldrich, $>99 \%$ ) and aluminum isopropoxide (Acros Organics $98 \%+$ ). The resulting mixture was aged at RT ( 4 days). After this a $51 \%$ HF solution (Acros organics, 48-51\%) was added and stirred into a homogeneous gel. The gel was transferred into three Teflon lined autoclaves, in equal portions. Autoclaves were sealed and put in a static oven at $150{ }^{\circ} \mathrm{C}$ for 6 days. After the synthesis was finished, the resulting solid was washed thoroughly with demineralized water $(\sim 8 \mathrm{~L})$. The resulting solid was a white powder. Calcination was performed in a static oven with the following temperature program: heat from room temperature to $150^{\circ} \mathrm{C}$ in $2 \mathrm{~h} 10 \mathrm{~min}$, hold for $1.5 \mathrm{~h}$, then heat to $350{ }^{\circ} \mathrm{C}$ over $1.5 \mathrm{~h}$ with a $3 \mathrm{~h}$ hold, then heat to $580^{\circ} \mathrm{C}$ over $4 \mathrm{~h} 50 \mathrm{~min}$ and hold for $3 \mathrm{~h}$ and finally cool to room temperature. Crystallinity was evaluated with XRD. The method used has been adapted from Lezcano-Gonzalez and colleagues ${ }^{3}$. The resulting $\mathrm{Si} / \mathrm{Al}$ ratio was $\sim 20$ as determined using ICP-OES. The $\mathrm{Cu}$ ion exchange was performed using $1 \mathrm{~g} \mathrm{SSZ}-13$ with $50 \mathrm{~mL}$ of a $0.1 \mathrm{M} \mathrm{CuSO}_{4} \cdot 5 \mathrm{H}_{2} \mathrm{O}$ (Merck ACS, ISO, Reag. Ph Eur) solution ( $\mathrm{pH}=4.3$ ) at $80^{\circ} \mathrm{C}$ for $2 \mathrm{~h}$. The resulting $\mathrm{Cu}-\mathrm{SSZ}-13$ material was washed with demineralized water and dried at $60^{\circ} \mathrm{C}$ overnight. Calcination was performed in a static oven with the following temperature program: heat from room temperature to $120^{\circ} \mathrm{C}$ in $1 \mathrm{~h}$, hold for $30 \mathrm{~min}$, then heat to $550^{\circ} \mathrm{C}$ over $7 \mathrm{~h} 10 \mathrm{~min}$ and hold for $4 \mathrm{~h}$, and finally cool to room temperature.

Zeolite ZSM-5 was purchased from Zeolyst (CBV2314, Lot 2200-89), with a specified $\mathrm{Si} / \mathrm{Al}$ ratio of 11.5. Before $\mathrm{Cu}$ exchange, the ZSM-5 was calcined in air ( $1 \mathrm{~K} \mathrm{~min}^{-1}$ to $120^{\circ} \mathrm{C}$, hold $30 \mathrm{~min}$, ramp $1 \mathrm{~K} \mathrm{~min}^{-1}$ to $550{ }^{\circ} \mathrm{C}$ and hold for $4 \mathrm{~h}$ ). The $\mathrm{Cu}$ ion exchange was conducted following the method of ref. ${ }^{52}$. A $10 \mathrm{mM}$ solution of $\mathrm{Cu}(\mathrm{II})$ acetate was used without $\mathrm{pH}$ adjustment, with $250 \mathrm{~mL}$ solution per gram catalyst, and the suspension was stirred at room temperature for $24 \mathrm{~h}$, and recovered using filtration and washed with a large excess of demineralized water. After drying, the material was recalcined in air using the same program.

Aging was conducted based on the industry standard simulation for a 135,000 mile vehicle-aged catalyst ${ }^{28}$. The catalyst was ramped to $800{ }^{\circ} \mathrm{C}$ at $2 \mathrm{~K} \mathrm{~min}^{-1}$ and held for $16 \mathrm{~h}$. The steaming was conducted using $10 \%$ steam in air flowing at $100 \mathrm{~mL} \mathrm{~min}^{-1}$, with the steam was created using a bubbler at $47^{\circ} \mathrm{C}$. Steam was first introduced when the catalyst reached $120^{\circ} \mathrm{C}$ to prevent condensation. The steaming was stopped prior to cooling the catalyst back to room temperature and was switched to dry air.

Catalyst testing. Catalytic activity tests were performed in a fixed bed plug flow set up. Typically, $50 \mathrm{mg}$ of powdered catalyst material (sieve fractions of $0.425-0.150 \mathrm{~mm}$ ) was loaded in a $1 \mathrm{~cm}$ OD quartz tubular reactor. Prior to the experiment, the zeolite sample was pre-treated for $1 \mathrm{~h}$ with $5 \% \mathrm{O}_{2}$ in $\mathrm{He}$ at $550{ }^{\circ} \mathrm{C}$. After the pre-treatment, the desired reaction temperature was fixed, and then the 
catalyst exposed to a SCR feed composition of $1000 \mathrm{ppm} \mathrm{NO}, 1000 \mathrm{ppm} \mathrm{NH}_{3}$ and 5 $\% \mathrm{O}_{2}$, and He for balance, with a Gas Hourly Space Velocity (GHSV) of 100,000 h ${ }^{-1}$. Steady-state measurements were performed at different reaction temperatures, from 150 to $450^{\circ} \mathrm{C}$, using a stabilization period of $60 \mathrm{~min}$ at each temperature and analyzing the output gases by mass spectrometry (Hiden Analytical, HPR-20 QIC) and FT-IR gas analysis. All SCR gases were provided by Linde. To avoid condensation in the reaction system, all the gas lines were heated to $150^{\circ} \mathrm{C}$. Equations 1 and 2 were used to calculate respectively the $\mathrm{NO}$ conversion and $\mathrm{N}_{2}$ selectivity.

$$
\begin{gathered}
\mathrm{NO} \text { conversion }(\%)=\frac{\mathrm{NO}_{\text {in }}-\mathrm{NO}_{\text {out }}}{\mathrm{NO}_{\text {in }}} \times 100 \\
\mathrm{~N}_{2} \text { selectivity }(\%)=\frac{\mathrm{NO}_{\text {in }}-\mathrm{NO}_{\text {out }}-\mathrm{N}_{2} \mathrm{O}_{\text {out }}-\mathrm{NO}_{2 \text { out }}}{\mathrm{NO}_{\text {in }}} \times 100
\end{gathered}
$$

Catalyst characterization. UV-Vis-NIR-DRS was collected using a Varian Cary 500 UV-Vis-NIR spectrometer equipped with a DRS accessory to allow collection in the diffuse reflectance mode, against a pure white reference standard. Spectra were collected between 4000 and $50,000 \mathrm{~cm}^{-1}$ with a data interval of $10 \mathrm{~cm}^{-1}$ and at a rate of $6000 \mathrm{~cm}^{-1} \mathrm{~min}^{-1}$. The UV-Vis-NIR-DRS spectra were corrected for the detector/grating and light source changeover steps at $11,400,12,500$, and $28,570 \mathrm{~cm}^{-1}$, respectively. Dehydrated, $\mathrm{O}_{2}$ activated samples were prepared under dry $\mathrm{O}_{2}$ flowing at $30 \mathrm{~mL} \mathrm{~min}^{-1}$ by heating to $450{ }^{\circ} \mathrm{C}$ at a rate of $5 \mathrm{~K} \mathrm{~min}^{-1}$, with a $2 \mathrm{~h}$ dwell before cooling to room temperature for measurement.

X-ray diffraction $(\mathrm{XRD})$ patterns were recorded on a Bruker D2 X-ray powder diffractometer equipped with a Co $K_{\alpha} \mathrm{X}$-ray tube $(\lambda=1.7902 \AA)$.

The chemical composition of the Cu-SSZ-13 zeolite sample has been determined with Optical Emission Spectroscopy (OES) after activation with Inductively Coupled Plasma (ICP). ICP-OES has been performed by the Geolab (Utrecht University), using a SPECTRO CIROSCCD (by SPECTRO Analytical Instruments $\mathrm{GmbH}-\mathrm{Germany})$. Samples were dissolved using an aqua regia with $\mathrm{HF}$ solution, in which they were dissolved at $90^{\circ} \mathrm{C}$ overnight, after which it was cooled down to RT and neutralized using boric acid. After this the solutions were diluted to yield to appropriate concentrations. The chemical composition of the Cu-ZSM-5 was measured using energy dispersive spectroscopy (EDS) with a EDAX SUTW Sapphire Detector in a Philips XL30 scanning electron microscope (SEM) at an accelerating voltage of $20 \mathrm{kV}$.

Temperature-programmed desorption of ammonia $\left(\mathrm{NH}_{3}-\mathrm{TPD}\right)$ was performed on a Micromeritics Autochem II 2920 equipped with a TCD detector. Prior to TPD, $0.1 \mathrm{~g}$ of catalyst was first out gassed in $\mathrm{He}$ for $1 \mathrm{~h}$ at $600^{\circ} \mathrm{C}$ with a heating ramp of $10^{\circ} \mathrm{C} \mathrm{min}^{-1}$. Ammonia was adsorbed at $100^{\circ} \mathrm{C}$ until saturated, followed by flushing with $\mathrm{He}$ for $120 \mathrm{~min}$ at $100^{\circ} \mathrm{C}$. The ammonia desorption was monitored using the TCD detector until $600{ }^{\circ} \mathrm{C}$ with a ramp of $5^{\circ} \mathrm{C} \min ^{-1}$, using a flow of $25 \mathrm{~mL} \mathrm{~min}^{-1}$.

SEM has been performed on a FEI Nova 200 Dual-Beam SEM/FIB located within the Center for Nanophase Materials Sciences (CNMS) at Oak Ridge National Laboratory (ORNL). The microscope is equipped with the following options: FEG scanning electron microscope, Ion column with Ga liquid ion source for milling, GIS for Pt deposition, Kleindiek nanomanipulator for specimen lift-out and AutoTEM, AutoFIB, and slice and view automation software.

Atom probe tomography. Needles for APT analysis were prepared from the four sets of sample under study, namely fresh Cu-ZSM- 5 and Cu-SSZ-13 as well as aged $\mathrm{Cu}-\mathrm{ZSM}-5$ and $\mathrm{Cu}-\mathrm{SSZ}-13$, using FIB milling. For this purpose, seven samples that yielded APT data sets are summarized in Supplementary Table 1. A representative set of SEM images of the APT needles are shown in Supplementary Fig. 6. For $\mathrm{Cu}-$ SSZ-13 the crystals were large enough $(\sim 4 \mu \mathrm{m})$ that standard lift-out and needle preparation were performed using standard specimen preparation techniques utilizing Si micro-tip arrays purchased from CAMECA ${ }^{53}$. For Cu-ZSM-5 the zeolite crystal aggregates were too small for standard preparation, so crystals were attached to the lift-out needle by electrostatic force and transferred to Si micro tips where they were FIB milled into needle-shaped specimens. The needle specimens were transferred to the LEAP 4000XR local electrode atom probe equipped with laser pulsing capabilities and an energy compensating reflectron lens located within the CNMS at ORNL. The specimens were run in laser pulse mode with a laser energy of $200 \mathrm{pJ}$, base temperature of $40 \mathrm{~K}$, pulse repetition rate of $200 \mathrm{kHz}$, and a detection rate of 1 atom per 200 pulses. The detector has an efficiency of $\sim 37 \%$. Data analysis is described in the supporting methods, following our previous publication $^{54}$. Videos of the APT sample reconstructions are available in the supporting information to aid with visualizing the ion distributions, clusters and isoconcentration surfaces in $3 \mathrm{D}$.

Data availability. All data are available from the corresponding authors on reasonable request.

Received: 11 May 2017 Accepted: 13 October 2017 Published online: 21 November 2017

\section{References}

1. Granger, P. \& Parvulescu, V. I. Catalytic NOx abatement systems for mobile sources: From three-way to lean burn after-treatment technologies. Chem. Rev. 111, 3155-3207 (2011).

2. Oord, R. \& Weckhuysen, B. M. in Zeolites and Zeolite-Like Materials (eds Sels, B. F. \& Kustov, L. M.) 433-450 (Elsevier, Amsterdam, 2016).

3. Beale, A. M., Lezcano-Gonzalez, I., Slawinksi, W. A. \& Wragg, D. S. Correlation between $\mathrm{Cu}$ ion migration behaviour and deNOx activity in Cu-SSZ-13 for the standard NH3-SCR reaction. Chem. Commun. 52, 6170-6173 (2016).

4. Moliner, M., Martínez, C. \& Corma, A. Synthesis strategies for preparing useful small pore zeolites and zeotypes for gas separations and catalysis. Chem. Mater. 26, 246-258 (2014).

5. Beale, A. M., Gao, F., Lezcano-Gonzalez, I., Peden, C. H. F. \& Szanyi, J. Recent advances in automotive catalysis for NOx emission control by small-pore microporous materials. Chem. Soc. Rev. 44, 7371-7405 (2015).

6. Iwamoto, M. et al. Copper(II) ion-exchanged ZSM-5 zeolites as highly active catalysts for direct and continuous decomposition of nitrogen monoxide. J. Chem. Soc. Chem. Commun. 1272-1273 (1986).

7. Kharas, K. C. C., Robota, H. J. \& Liu, D. J. Deactivation in Cu-ZSM-5 lean-burn catalysts. Appl. Catal. B Environ. 2, 225-237 (1993).

8. Yan, J. Y., Lei, G.-D., Sachtler, W. M. H. \& Kung, H. H. Deactivation of Cu/ ZSM-5 catalysts for lean NOx reduction: Characterization of changes of $\mathrm{Cu}$ state and zeolite support. J. Catal. 161, 43-54 (1996).

9. Brandenberger, S., Kröcher, O., Tissler, A. \& Althoff, R. The state of the art in selective catalytic reduction of NOx by Ammonia using metal-exchanged zeolite catalysts. Catal. Rev. 50, 492-531 (2008).

10. Bull, I. et al. Copper CHA zeolite catalysts. US patent 7,601,662 B2 (2009).

11. Göltl, F., Bulo, R. E., Hafner, J. \& Sautet, P. What makes copper-exchanged SSZ-13 zeolite efficient at cleaning car exhaust gases? J. Phys. Chem. Lett. 4, 2244-2249 (2013).

12. Kwak, J. H., Tonkyn, R. G., Kim, D. H., Szanyi, J. \& Peden, C. H. F. Excellent activity and selectivity of Cu-SSZ-13 in the selective catalytic reduction of NOx with NH3. J. Catal. 275, 187-190 (2010).

13. Tian, P., Wei, Y., Ye, M. \& Liu, Z. Methanol to olefins (MTO): from fundamentals to commercialization. ACS Catal. 5, 1922-1938 (2015).

14. Deka, U. et al. Confirmation of Isolated $\mathrm{Cu} 2+$ ions in SSZ-13 zeolite as active sites in NH3-selective catalytic reduction. J. Phys. Chem. C 116, 4809-4818 (2012).

15. Wang, J., Zhao, H., Haller, G. \& Li, Y. Recent advances in the selective catalytic reduction of NOx with $\mathrm{NH} 3$ on Cu-Chabazite catalysts. Appl. Catal. B Environ. 202, 346-354 (2017).

16. Paolucci, C., Di Iorio, J. R., Ribeiro, F. H., Gounder, R. \& Schneider, W. F. Advances in Catalysis Vol. 59, 1-107 (Elsevier, Amsterdam, 2016).

17. Paolucci, C. et al. Catalysis in a cage: condition-dependent speciation and dynamics of exchanged Cu cations in SSZ-13 zeolites. J. Am. Chem. Soc. 138, 6028-6048 (2016).

18. Lezcano-Gonzalez, I. et al. Determining the storage, availability and reactivity of $\mathrm{NH}_{3}$ within $\mathrm{Cu}$-Chabazite-based ammonia selective catalytic reduction systems. Phys. Chem. Chem. Phys. 16, 1639-1650 (2014).

19. Godiksen, A. et al. Coordination environment of copper sites in $\mathrm{Cu}-\mathrm{CHA}$ zeolite investigated by electron paramagnetic resonance. J. Phys. Chem. C 118, 23126-23138 (2014)

20. Bates, S. A. et al. Identification of the active $\mathrm{Cu}$ site in standard selective catalytic reduction with ammonia on Cu-SSZ-13. J. Catal. 312, 87-97 (2014).

21. Deka, U., Lezcano-Gonzalez, I., Weckhuysen, B. M. \& Beale, A. M. Local environment and nature of $\mathrm{Cu}$ active sites in zeolite-based catalysts for the selective catalytic reduction of NOx. ACS Catal. 3, 413-427 (2013).

22. Luo, J. et al. Identification of two types of $\mathrm{Cu}$ sites in Cu/SSZ-13 and their unique responses to hydrothermal aging and sulfur poisoning. Catal. Today 267, 3-9 (2016).

23. Wang, D. et al. A comparison of hydrothermal aging effects on NH3-SCR of NOx over Cu-SSZ-13 and Cu-SAPO-34 catalysts. Appl. Catal. B Environ. 165 438-445 (2015).

24. Su, W., Li, Z., Peng, Y. \& Li, J. Correlation of the changes in framework and active $\mathrm{Cu}$ sites for typical $\mathrm{Cu} / \mathrm{CHA}$ zeolite (SSZ-13 and SAPO-34) during hydrothermal aging. Phys. Chem. Chem. Phys. 17, 29142-29149 (2015).

25. Wang, J. et al. Influence of aging on in situ hydrothermally synthesized CuSSZ-13 catalyst for NH3-SCR reaction. RSC Adv. 4, 42403-42411 (2014).

26. Ma, L. et al. Characterization of commercial Cu-SSZ-13 and Cu-SAPO-34 catalysts with hydrothermal treatment for NH3-SCR of NOx in diesel exhaust. Chem. Eng. J. 225, 323-330 (2013).

27. Kwak, J. H. et al. Effects of hydrothermal aging on NH3-SCR reaction over $\mathrm{Cu}$ / zeolites. J. Catal. 287, 203-209 (2012).

28. Schmieg, S. J. et al. Thermal durability of Cu-CHA NH3-SCR catalysts for diesel NOx reduction. Catal. Today 184, 252-261 (2012).

29. Fickel, D. W., D'Addio, E., Lauterbach, J. A. \& Lobo, R. F. The ammonia selective catalytic reduction activity of copper-exchanged small-pore zeolites. Appl. Catal. B Environ. 102, 441-448 (2011). 
30. Vennestrøm, P. N. R. et al. Migration of $\mathrm{Cu}$ ions in SAPO-34 and its impact on selective catalytic reduction of NOx with NH3. ACS Catal. 3, 2158-2161 (2013).

31. Groothaert, M. H., van Bokhoven, J. A., Battiston, A. A., Weckhuysen, B. M. \& Schoonheydt, R. A. Bis( $\mu$-oxo)dicopper in Cu-ZSM-5 and its role in the decomposition of NO: a combined in situ XAFS, UV-Vis-Near-IR, and kinetic study. J. Am. Chem. Soc. 125, 7629-7640 (2003).

32. Deka, U. et al. Changing active sites in $\mathrm{Cu}-\mathrm{CHA}$ catalysts: deNOx selectivity as a function of the preparation method. Microporous Mesoporous Mater. 166, 144-152 (2013).

33. Wang, L., Gaudet, J. R., Li, W. \& Weng, D. Migration of $\mathrm{Cu}$ species in $\mathrm{Cu} /$ SAPO-34 during hydrothermal aging. J. Catal. 306, 68-77 (2013).

34. Vennestrøm, P. N. R. et al. Influence of lattice stability on hydrothermal deactivation of Cu-ZSM- 5 and Cu-IM- 5 zeolites for selective catalytic reduction of NOx by NH3. J. Catal. 309, 477-490 (2014).

35. Blakeman, P. G. et al. The role of pore size on the thermal stability of zeolite supported Cu SCR catalysts. Catal. Today 231, 56-63 (2014).

36. Valdez Lancinha Pereira, M., Nicolle, A. \& Berthout, D. Hydrothermal aging effects on Cu-zeolite NH3-SCR catalyst. Catal. Today 258, 424-431 (2015).

37. Shwan, S. et al. Solid-state ion-exchange of copper into zeolites facilitated by ammonia at low temperature. ACS Catal. 5, 16-19 (2015).

38. Wang, L., Li, W., Qi, G. \& Weng, D. Location and nature of $\mathrm{Cu}$ species in $\mathrm{Cu} /$ SAPO-34 for selective catalytic reduction of NO with NH3. J. Catal. 289, 21-29 (2012).

39. Kovarik, L. et al. Transformation of active sites in Fe/SSZ-13 SCR catalysts during hydrothermal aging: a spectroscopic, microscopic, and kinetics study. ACS Catal. 7, 2458-2470 (2017).

40. Perea, D. E. et al. Determining the location and nearest neighbours of aluminium in zeolites with atom probe tomography. Nat. Commun. 6, 7589 (2015).

41. Liu, K. et al. Rationalizing inter- and intracrystal heterogeneities in dealuminated acid mordenite zeolites by stimulated Raman scattering microscopy correlated with super-resolution fluorescence microscopy. ACS Nano 8, 12650-12659 (2014).

42. Van Loon, J. et al. Rationalizing acid zeolite performance on the nanoscale by correlative fluorescence and electron microscopy. ACS Catal. 7, 5234-5242 (2017).

43. Kubarev, A. V., Janssen, K. P. F. \& Roeffaers, M. B. J. Noninvasive nanoscopy uncovers the impact of the hierarchical porous structure on the catalytic activity of single dealuminated mordenite crystals. ChemCatChem 7, 3646-3650 (2015).

44. Fodor, D., Krumeich, F., Hauert, R. \& Van Bokhoven, J. A. Differences between individual ZSM-5 crystals in forming hollow single crystals and mesopores during base leaching. Chem. Eur. J. 21, 6272-6277 (2015).

45. Martin, A. J., Mitchell, S., Kunze, K., Weston, K. C. \& Pérez-Ramírez, J. Visualising compositional heterogeneity during the scale up of multicomponent zeolite bodies. Mater. Horiz. 4, 857-861 (2017).

46. Chen, Y., Chou, P. H. \& Marquis, E. A. Quantitative atom probe tomography characterization of microstructures in a proton irradiated 304 stainless steel. $J$. Nucl. Mater. 451, 130-136 (2014).

47. Cairney, J. M. et al. Mining information from atom probe data. Ultramicroscopy 159, 324-337 (2015).

48. Shishkin, A., Kannisto, H., Carlsson, P.-A., Härelind, H. \& Skoglundh, M. Synthesis and functionalization of SSZ-13 as an NH3-SCR catalyst. Catal. Sci. Technol. 4, 3917-3926 (2014).

49. Clemens, A. K. S. et al. Reaction-driven ion exchange of copper into Zeolite SSZ-13. ACS Catal. 5, 6209-6218 (2015).

50. Albarracin-Caballero, J. D. et al. Structural and kinetic changes to small-pore $\mathrm{Cu}$-zeolites after hydrothermal aging treatments and selective catalytic reduction of NOx with ammonia. React. Chem. Eng. 2, 168-179 (2017).

51. Paolucci, C. et al. Dynamic multinuclear sites formed by mobilized copper ions in NOx selective catalytic reduction. Science 357, 898-903 (2017).

52. Giordanino, F. et al. Characterization of $\mathrm{Cu}$-exchanged SSZ-13: a comparative FTIR, UV-Vis, and EPR study with Cu-ZSM- 5 and $\mathrm{Cu}-\beta$ with similar Si/Al and $\mathrm{Cu} / \mathrm{Al}$ ratios. Dalton Trans. 42, 12741-12761 (2013).
53. Thompson, K. et al. In situ site-specific specimen preparation for atom probe tomography. Ultramicroscopy 107, 131-139 (2007)

54. Schmidt, J. E. et al. Coke formation in a zeolite crystal during the methanol-tohydrocarbons reaction as studied with atom probe tomography. Angew. Chem. Int. Ed. 55, 11173-11177 (2016).

55. Miller, M. K. Atom Probe Tomography (Springer, Boston, MA, 2000).

\section{Acknowledgements}

This work is supported by the NWO Gravitation program, Netherlands Center for Multiscale Catalytic Energy Conversion (MCEC), and a European Research Council (ERC) Advanced Grant (No. 321140). The APT measurements were conducted at the Center for Nanophase Materials Sciences, which is a DOE Office of Science User Facility. J.S. has received funding from the European Union's Horizon 2020 research and innovation program under the Marie Sklodowska-Curie Grant Agreement No. 702149. This manuscript has been authored by UT-Battelle, LLC under Contract No. DE-

AC05-00OR22725 with the U.S. Department of Energy. The United States Government retains and the publisher, by accepting the article for publication, acknowledges that the United States Government retains a non-exclusive, paid-up, irrevocable, world-wide license to publish or reproduce the published form of this manuscript, or allow others to do so, for United States Government purposes. The Department of Energy will provide public access to these results of federally sponsored research in accordance with the DOE Public Access Plan (http://energy.gov/downloads/doe-public-access-plan).

\section{Author contributions}

J.E.S., R.O., J.D.P., and B.M.W. conceived the project, designed the experiments, and interpreted the data. J.E.S. and R.O. synthesized and characterized the materials R.O. measured UV-Vis-NIR-DRS spectra, $\mathrm{NH}_{3}-\mathrm{TPD}$, and $\mathrm{NO}_{X}$ reduction reactions. J.E.S, W.G., and J.D.P. prepared samples for APT, collected, and analyzed the APT data. All authors participated in the writing of the manuscript.

\section{Additional information}

Supplementary Information accompanies this paper at doi:10.1038/s41467-017-01765-0.

Competing interests: The authors declare no competing financial interests.

Reprints and permission information is available online at http://npg.nature.com/ reprintsandpermissions/

Publisher's note: Springer Nature remains neutral with regard to jurisdictional claims in published maps and institutional affiliations.

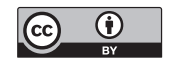

Open Access This article is licensed under a Creative Commons Attribution 4.0 International License, which permits use, sharing, adaptation, distribution and reproduction in any medium or format, as long as you give appropriate credit to the original author(s) and the source, provide a link to the Creative Commons license, and indicate if changes were made. The images or other third party material in this article are included in the article's Creative Commons license, unless indicated otherwise in a credit line to the material. If material is not included in the article's Creative Commons license and your intended use is not permitted by statutory regulation or exceeds the permitted use, you will need to obtain permission directly from the copyright holder. To view a copy of this license, visit http://creativecommons.org/ licenses/by/4.0/.

(C) The Author(s) 2017 